\section{True genius}

Oral-B's Genius brush, combined with the Oral-B App, provides the world's most intelligent brushing system, helping users to achieve the best possible at-home dental care.

It does this by combining the best cleaning technology - electric tooth-brushing - with the best guiding technology. Oral-B's oscillating-rotating-pulsating brush-heads provide an outstanding clean, while Oral-B’s App makes sure that users brush for the right amount of time, with the right pressure, and that all zones of the mouth are covered evenly. Even the best cleaning and brushing technology in the world cannot help if it isn't applied everywhere evenly.

The position detection capability of the Oral-B Genius is what sets it apart. Other brushes cannot detect where users brush in their mouth as Genius can. Their systems give recommendations on where to brush, but cannot track if indeed the user brushes where their app tells them to do. If the user does not follow the guidance given on the app-screen, their brushes cannot detect where the

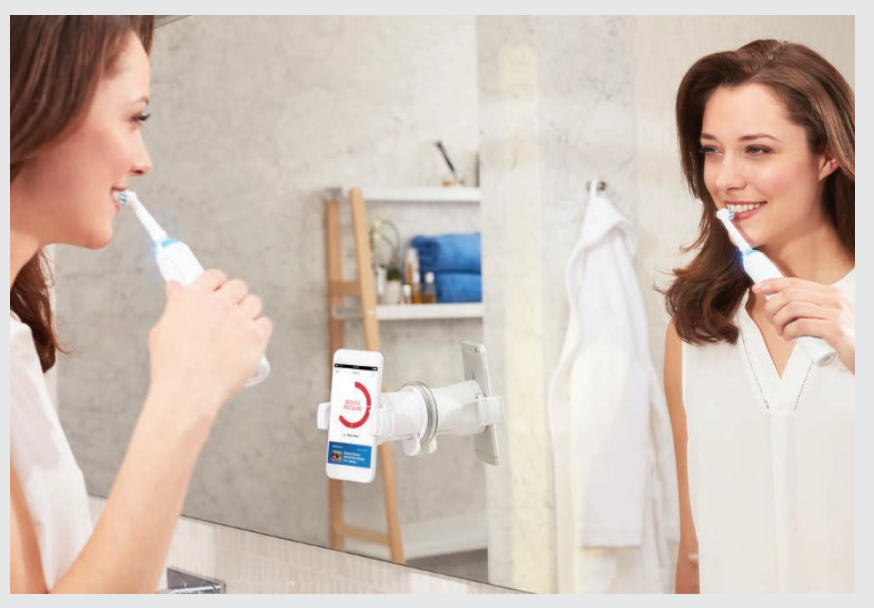

brush is, and cannot help the user to brush correctly, or to improve their brushing style.

Genius? It certainly is!

\title{
Universal adhesive with active moisture control
}

Designed for all etching methods and indications, Prime\&Bond active from Dentsply Sirona features patented Active-Guard Technology - a significant advancement in adhesive chemistry that actively controls moisture on the prepared surface. Even if dentine is overly wet or dry, Prime\&Bond active is simple to use and provides consistent results for a strong, reliable bond.

Most dental adhesives are rather hydrophobic: they separate from water. With too much water on the prepared surface, these rather hydrophobic adhesives fail to cover the prepared surface uniformly. During air-drying, adhesive gaps can form as embedded pockets of water evaporate, increasing the risks of post-operative sensitivity and bond failure. Active-Guard Technology with active moisture control is exclusively designed to protect against these risks.

The Active-Guard Technology balances hydrophobic and hydrophilic features and helps to achieve an optimised surface tension. As a result, Prime\&Bond active can overcome the surface tension of water, allowing the adhesive to spread evenly across the dentine and into the dentinal tubules to form a uniform, homogeneous layer. When the adhesive is air-dried, solvent and excess water evaporate uniformly to leave a thin, consistent coating of adhesive across the entire surface. The result is a strong, reliable bond, with virtually no post-operative sensitivity.

In addition to active moisture control, Prime\&Bond active provides several benefits over competing universal adhesives, including:

- Active spreading across the prepared surface to minimise the risk of dry spots that can lead to post-operative sensitivity and micro-leakage

- Low film thickness, minimising the risks of changing the preparation geometry and compromising crown fit, or leaving adhesive pools that may show up on radiographs and can be misdiagnosed a voids, gaps or secondary decay

- Convenience and control, with one-handed opening of Flip Top Cap bottle, precise dispensing and up to 30 minutes of working time in a closed CliXdish.

- Innovative formulation without HEMA, TGDMA and Bisphenol.

To find out more about the extensive range of restorative solutions,

\section{The ethical way to treat patients}

Using nickel titanium coil springs to guide patients' teeth into position, the ClearSmile Inman Aligner is a safe and ethical way to treat simple to moderate cases of misaligned, crowded or protruding anterior teeth.

The appliance is also extremely predictable thanks to the unique combination of the Spacewize+ crowding calculator and Archwize planning software provided by IAS Academy as part of the treatment pathway. When used effectively along with careful case selection and planning, the optimal treatment outcome can be achieved for your patient, every time.

To offer the ClearSmile Inman Aligner to your patients, you simply need to complete the IAS Academy's in-depth course, followed by the submission of two cases via the online support forum.

To find out more about training available near you, visit http://www.iasortho.com/courses.html. For more information on upcoming IAS Academy training courses, visit www.iasortho.com or call 02089162024. 